\title{
Influence of antiplatelet-anticoagulant drugs on the need of blood components transfusion after vesical transurethral resection
}

\author{
Alvaro Julio Virseda-Rodríguez ${ }^{1}$, Barbara Padilla-Fernández ${ }^{2}$, Mirian López-Parra ${ }^{3}$ \\ Maria Tatiana Santos-Antunes ${ }^{4}$, Lauro Sebastian Valverde-Martínez ${ }^{1}$, Maria Jesus Nieto-González ${ }^{3}$, \\ Jesus Fernando San Miguel-Izquierdo ${ }^{5}$, Anabel Lorenzo-Gómez ${ }^{4}$, Maria Begoña García-Cenador ${ }^{4}$, \\ Patricia Antúnez-Plaza ${ }^{6}$, Maria Fernanda Lorenzo-Gómez ${ }^{1,4}$ \\ ${ }^{1}$ Department of Urology, University Hospital of Salamanca; \\ 2 Department of Urology, University Hospital of the Canary Islands (Tenerife); \\ ${ }^{3}$ Department of Haematology, University Hospital of Salamanca; \\ ${ }^{4}$ Department of Surgery, University of Salamanca; \\ ${ }^{5}$ Clinical and Translational Medicine of the University of Navarra; \\ ${ }^{6}$ Department of Pathology, University Hospital of Salamanca; \\ University Hospital of Salamanca. Surgery Department of the University of Salamanca. IBSAL (Instituto de Investigación Biomédica \\ de Salamanca).
}

\begin{abstract}
Summary Aims: The effect of the antithrombotic preventive therapy on haemorrhage keeps uncertain. We investigate the influence of the antiplatelet and anticoagulant drugs (AP/AC drugs) on the transfusion requirement after vesical transurethral resection (VTUR). We also describe the epidemiology of the blood components transfusion in our department.

Materials and Methods: Retrospective observational study of a series of patients needing blood transfusion at the Urology Department between June 2010 and June 2013. Selection of 100 consecutive patients who were transfused after VTUR due to bladder transitional cell carcinoma (BTCC) (group A = GA). Control group: 100 consecutive patients who underwent VTUR due to BTCC and were not transfused (group $B=G B$ ).

Transfusion criteria: Haemoglobin $<8 \mathrm{~g} / \mathrm{dl}+$ anaemia symptoms. Age, gender, associated AP/AC treatment, secondary diagnoses, toxics, tumour stage and grade were analysed. Results: 212 patients required transfusion of a blood component. 169 were men (79\%) and 43 women (21\%). Median age 77.59 years (SD 9.42, range 50-92). Secondary diagnoses: Diabetes Mellitus 64\%, high blood pressure $77 \%$, dyslipidemia $52 \% .60 \%$ of patients were previously treated with AP/AC drugs. Average Haemoglobin pre-transfusion values: $7.4 \mathrm{~g} / \mathrm{dl}(\mathrm{DE} \pm 0.7)$. Average Haemoglobin post-transfusion values: $8.9 \mathrm{~g} / \mathrm{Dl}(\mathrm{DE} \pm$ 0.72 ). Most frequent transfusion indications were bladder cancer (37\%), kidney cancer (11\%), prostate cancer (8\%), benign prostatic hyperplasia (BHP) (8\%), other urological diagnoses (36\%). Intraoperative transfusions indicated by the anaesthesiologist: kidney cancer (33\%), BPH (28\%).

Patients who underwent VTUR due to BTCC were older in GA (77.59 years $S D$ 9.42) than in GB (68.98 years $S D 11.78)(p=$ 0.0001 ). Similar gender distribution (15 women in GA and 24 in GB). Less patients were asked to keep their treatment with ASA 100mg (AcetylSalicylicAcid) in GA (25.64\%) than in GB $(50 \%)(p=0.0330)$. More aggressive tumour grade in $G A(p=$ $0.0003)$ and higher stage in GA $(p=0.0018)$ regardless of concomitant treatment with AP/AC drugs.

Conclusions: The pathologies which most needed blood components' transfusions in the Urology Department were (in order of frequency): bladder cancer, kidney cancer, prostate cancer, prostate adenoma. ASA100mg did not influence the transfu-
\end{abstract}

sion's requirements in VTUR due to BTCC. Tumour stage and higher grade have a greater influence in transfusion's requirements than concomitant AP/AC treatment. The heterogeneity of AP/AC protocols does not allow to establish the benefit of stopping those drugs before surgery in terms of avoiding blood transfusions when performing a VTUR.

KEY WORDS: Transurethral resection; Bladder transitional carcinoma; Blood transfusion.

Submitted 23 December 2014; Accepted 28 January 2015

\section{INTRODUCTION}

The urologic surgery is considered as having a high haemorrhagic risk. The development of several blood components in the last decades has changed the management of multiple pathologies and has increased the survival of millions of patients.

Haematuria with anaemia which requires blood transfusion is considered a surgical complication in the recording codes of the hospital processes. There are in the market several antiplatelet and anticoagulant drugs (AP/AC) which are frequently part of the regular treatment of many urological patients. Other specialists (Haematologists, Cardiologists, Anaesthesiologists,...) prescribe withdrawal and reintroduction standards when a vesical transurethral resection (VTUR) is required. We investigated the factors which could condition the transfusion requirement in patients taking AP/AC drugs.

Aims of the study were: to describe the number of transfusions performed in a "standard" Urology department attending to 333,000 inhabitants and their indication and to investigate the risk factors related with the blood transfusion requirements in patients who underwent VTUR due to bladder transitional cell carcinoma (BTCC). 


\section{Materials AND METHOdS}

In order to reach the aims of the study, we have selected two series of patients and carried out two independent retrospective observational studies:

- Series of patients needing blood transfusion at the Urology Department between June 2010 and June 2013.

- Selection of 100 consecutive patients who were transfused after VTUR due to bladder transitional cell carcinoma (BTCC) (group A = GA) between June 2008 and June 2013. Control group: 100 consecutive patients who underwent VTUR due to BTCC and were not transfused (group B $=$ GB) at the same period.

The same transfusion criteria are applied for all patients: Haemoglobin $<8 \mathrm{~g} / \mathrm{dl}+$ anaemia symptoms. Age, gender, associated AP/AC treatment, second diagnoses, toxics, tumour stage and grade were analysed. Descriptive statistics, Student's t-test, Fisher's exact test, ANOVA were utilized and $p<0.05$ was considered significant.

\section{Results}

212 patients required transfusion of a blood component when admitted at the Urology department during the period of the study. 169 were men $(79 \%)$ and 43 women (21\%). Median age 77.59 years (SD 9.42, range 50-92). Secondary diagnoses: Diabetes Mellitus 64\%, high blood pressure 77\%, dyslipidemia $52 \% .60 \%$ of patients were previously treated with AP/AC drugs. Transfusion was indicated with an average Haemoglobin pre-transfusion value of $7.4 \mathrm{~g} / \mathrm{dl}(\mathrm{DE} \pm 0.7)$. The average Haemoglobin posttransfusion value was $8.9 \mathrm{~g} / \mathrm{dl}(\mathrm{DE} \pm 0.72)$. The most frequent transfusion indication was bladder cancer $(37 \%)$, followed by kidney cancer (11\%). Prostate cancer and benign prostatic hyperplasia (BPH) share the third place (8\% each) (Figure 1).

Table 1 shows the distribution of those indications which required blood components' transfusion at the Urology department (Table 1).

Most intraoperative transfusions indicated by the anaesthesiologist were due to kidney cancer (33\%) and BPH (28\%) (Table 2).

\section{Figure 1.}

Main diagnosis in patients who required blood transfusion.

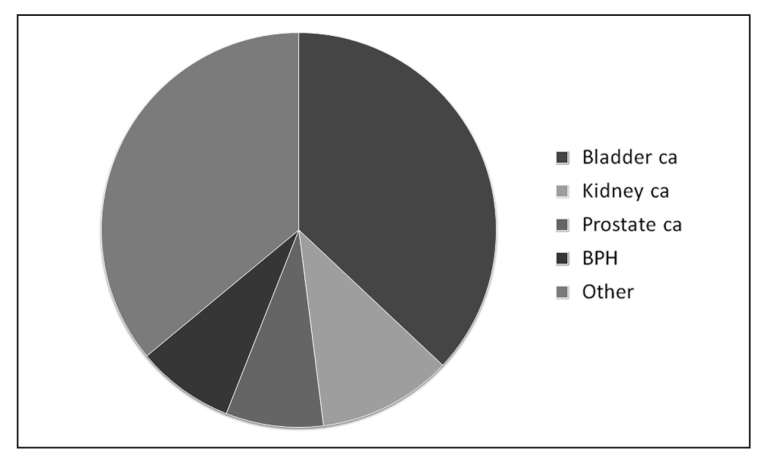

Table 1.

Distribution of those indications which required blood components' transfusion at the Urology department.

\begin{tabular}{|l|cccccc|}
\hline & N & $\%$ & M & $\%$ & W & $\%$ \\
\hline Bladder cancer & 79 & 37.3 & 55 & 68 & 24 & 32 \\
Kidney cancer & 24 & 11.9 & 13 & 54 & 11 & 46 \\
Prostate cancer & 17 & 8.7 & 17 & 100 & 0 & 0 \\
BPH & 17 & 8.7 & 17 & 100 & 0 & 0 \\
Stones & 3 & 1.1 & 1 & 33 & 2 & 66 \\
Intra abdominal abscess & 3 & 1.1 & 2 & 66 & 1 & 33 \\
Urinary tract cysts & 2 & 0.94 & 1 & 50 & 1 & 50 \\
Haematuria as admission diagnosis & & & & & & \\
at the Emergency department & 36 & 16.98 & 23 & 63.1 & 13 & 36.9 \\
Gross haematuria at the Emergency & & & & & & \\
department with concomitant AP/AC & 27 & 74.9 & 14 & 53.1 & 13 & 46.9 \\
\hline With final diagnosis after discharged at the first admission & 19 & 52.3 & 13 & 64.2 & 6 & 35.8 \\
\hline Total & 9 & 25 & 7 & 77 & 2 & 23 \\
Bladder cancer & 4 & 11 & 4 & 100 & & \\
BPH & 3 & 8.3 & 2 & 66.6 & 1 & 33.4 \\
Kidney cancer & 3 & 8.3 & 2 & 66.6 & 1 & 33.4 \\
Other & 13 & 76.2 & 7 & 54 & 6 & 46 \\
\hline Without final diagnosis after discharged at the first admission & & & \\
\hline Total & 17 & 47.7 & 9 & 52 & 8 & 48 \\
Anticoagulated & 13 & 1 & 33 & 3 & 66 \\
Not-anticoagulated & 4 & & & & \\
\hline M: men; W: women. & \multicolumn{7}{|c|}{23.8} \\
\hline
\end{tabular}

Table 2.

Surgeries with transfusion requirements.

\begin{tabular}{|l|cccccc|}
\hline & N & $\%$ & M & $\%$ & W & $\%$ \\
\hline Total & 39 & 18.2 & 28 & 70.8 & 10 & 29.2 \\
\hline Kidney cancer & 13 & 33.3 & 7 & 54.3 & 6 & 45.7 \\
\hline BPH & 11 & 28.1 & 11 & 100 & & \\
\hline Bladder cancer & 6 & 15.3 & 4 & 66.6 & 2 & 33.4 \\
\hline Kidney rupture, cystic pathology & 2 & & & & & \\
\hline Stones, kidney trasplantation, others & 2 & & & & & \\
\hline
\end{tabular}

Table 2.

Concomitant AP/AC drugs in patients undergoing VTUR.

\begin{tabular}{|l|c|c|c|}
\hline AP/AC & Group A (\%) & Group B (\%) & Signification \\
\hline Warfarin & 33.33 & 31.58 & 0.7215 \\
ASA 100 & 25.64 & $\mathbf{5 0}$ & $\mathbf{0 . 0 3 3 0}$ \\
ASA 300 & 7.69 & 7.89 & 0.6728 \\
Enoxaparin & 7.69 & 0 & 0.0938 \\
Pentoxifilin & 7.69 & 0 & 0.1692 \\
Clopidogrel & 5.13 & 10.25 & 0.4309 \\
Bemiparin & 5.13 & 0 & 0.7152 \\
Diosmin+Hesperidin deriv & 5.13 & 0 & 0.6430 \\
Trifusal & 2.56 & 0 & 0.4810 \\
\hline
\end{tabular}


Table 4.

Distribution of the tumour stage and grade in patients with or without AP/AC drugs who underwent VTUR due to BTCC.

\begin{tabular}{|l|cc|cc|c|}
\hline & \multicolumn{2}{|c|}{ Group A } & \multicolumn{2}{c|}{ Group B } & \multirow{2}{*}{ Signification } \\
& (-) AP/AC & (+) AP/AC & (-) AP/AC & (+) AP/AC & \\
\hline pTa & 13.72 & 20.68 & 35.41 & 22.22 & 0.4271 \\
pT1 & 15.68 & 13.79 & 33.33 & 38.88 & 0.6109 \\
pT2 & 45.09 & 41.37 & 16.66 & 13.88 & 0.0815 \\
pT3 & 17.64 & $\mathbf{2 0 . 6 8}$ & 4.16 & 5.55 & $\mathbf{0 . 0 0 1 8}$ \\
pT4 & 7.84 & 3.45 & 2.08 & & $\mathbf{0 . 0 0 0 4}$ \\
\hline G1 & 3.92 & 3.45 & 18.75 & 13.88 & 0.6182 \\
G2 & 1.96 & 10.34 & 35.41 & 30.55 & 0.7151 \\
G3 & $\mathbf{4 9 . 0 2}$ & 37.93 & 27.08 & 30.55 & $\mathbf{0 . 0 0 0 3}$ \\
\hline
\end{tabular}

Those patients who underwent VTUR due to BTCC were older in GA (77.59 years SD 9.42) than in GB (68.98 years SD 11.78) $(\mathrm{p}=0.0001)$. Similar gender distribution (15 women in GA and 24 in GB). The usual AP/AC treatment of patients admitted for VTUR due to BTCC was investigated (Table 3). Acetylsalicylic acid (ASA) 100 mg was not removed.

Table 4 shows the tumour grade and stage of the BTCC operated.

\section{Discussion}

Patients who undergo urological surgery are frequently treated with AP/AC drugs. Withdrawal and reintroduction standards are varied and must be adjusted depending on several risk factors and the characteristics of each patient. The effects of the antithrombics prophylaxis remains uncertain (1). In some meta-analysis, main or total haemorrhage is increased with the use of antiplatelet-anticoagulant drugs (AP/AC), but in other studies no increase was found (1).

Major surgery is associated with an important blood loss which involves a high morbidity and mortality (2). Preoperative evaluation is essential for the blood transfusion reduction and requires a detailed medical record, physical examination, previous family or personal haemorrhagic events and a list of AP/AC drugs which may be withdrawn or substituted prior to surgery (3).

\section{Acetylsalicylic acid (ASA)}

Irreversible inactivation of the cyclooxygenase enzyme required for prostaglandin and thromboxane synthesis $(4,5)$. The elimination half-life depends on the dose ingested, but its action duration does not have a direct relationship with the plasmatic half-life because the biotransformation pathways concerned with the formation of salicyluric acid and salicyl phenolic glucuronide becomes saturated (6). Oral administration is rather selective to platelets due to its pre-systemic withdrawal $(7,8)$. After ASA administration, the TXA2 production is not recovered until the platelets cohort has been substituted in 7-10 days $(7,8)$.

Clinical essays have shown the efficacy of ASA in several situations with similar efficacy in a dose range of 50-
$1500 \mathrm{mg} /$ day; those doses inhibited nearly completely the thromboxane synthesis in platelets (8). Nevertheless, the ASA's side-effects have a clear relationship with the dose, recommending a low dose for thrombotic prophylaxis (75 mg per day) (9). With very high doses, ASA also has an inhibitory effect on the haemostasis depending on vitamin $\mathrm{K}$, modifying the prothrombin production and developing an hypoprothrombinemia (9).

We did not find a higher bleeding risk in patients usually treated with ASA $300 \mathrm{mg}(\mathrm{p}=0.6728)$ after a preoperative withdrawal of 6-10 days.

When ASA $100 \mathrm{mg}$ was taken preoperatively, a higher bleeding proportion was found in GB ( $p=0.0330)$.

\section{Clopidogrel}

It is an oral, thienopyridine class antiplatelet agent with a similar structure and mechanism of action to ticlopidine (7). It works by irreversibly inhibiting the P2Y12 receptor, an adenosine diphosphate (ADP) chemoreceptor on platelet cell membranes (10). It is more effective than ASA reducing the number of atherosclerotic events in people on high risk (11). It has a similar tolerability to ASA, but clopidogrel seems to develop more severe neutropenia events (7). The drug is rapidly absorbed after oral administration of repeated doses of 75-milligram clopidogrel (min. 8 days), with peak plasma levels (approx. $3 \mathrm{mg} / \mathrm{L}$ ) of the main circulating metabolite occurring approximately one hour after dosing (12). When clopidogrel administration is stopped, its effect takes 5 more days to disappear (13).

Its main side-effect is haemorrhage. It is recommended to withdrawn clopidogrel 5 days before major surgery in order to avoid excessive bleeding (11). It has been reported an haemorrhage global incidence for patients treated with clopidogrel and ASA of $9.3 \%$, with an incidence of severe cases of $1.4 \%$ for clopidogrel and $1.6 \%$ for ASA (11). For patients taking clopidogrel, the incidence of gastrointestinal bleeding observed was $2 \%$, and a $0.7 \%$ required admission. For patients taking ASA, the corresponding percentages were $2.7 \%$ and $1.1 \%$, respectively (11). The incidence of other bleeding was higher in the clopidogrel group compared with ASA (7.3\% versus $6.5 \%)$. Nevertheless, the incidence of severe side-effects was similar in both treatment groups $(0.6 \%$ versus $0.4 \%)$. Most frequently reported side-effects with both treatments were: purpura, ecchymosis, hematoma and epistaxis (nosebleed). Other less frequent reported sideeffects were: haematuria and red eye (subconjunctival haemorrhage). The incidence of intracranial hemorrhage was of $0.4 \%$ in patients receiving clopidogrel and of $0.5 \%$ with ASA (11).

Concomitant administration of clopidogrel and naproxen increased the fecal occult blood. Nevertheless, more studies are needed to know if the increase of gastrointestinal bleeding is applicable to other nonsteroidal antiinflammatory drugs (NSAIDs) (14).

In our series, the clopidogrel withdrawal standard is given by the Anaesthesiologist at the preoperative study, varying between 7 and 12 days prior to surgery. With this withdrawal standard, we did not find a greater proportion of patients in group A with previous treatment with clopidogrel. 


\section{Acenocoumarol}

It is an anticoagulant that functions as a vitamin $\mathrm{K}$ antagonist. The peak level is reached 1-3 hours after its administration. It has an average half-life of 9 hours. Prothrombin time is restored to normal in a few days after withdraw. Its plasmatic elimination half-life is 8-11 hours: only an $0.12-0.18 \%$ is excreted unchanged in urine. The cumulative excretion of metabolites and unchanged active drug during 8 days reaches a $60 \%$ in urine and a 29\% in stool (15).

Due to interindividual changes, no correlation between the acenocoumarol plasma concentration and the prothrombin level can be made (15). Elderly patients (> 70 years old) usually have higher plasma concentrations than young patients with the same daily dose (15). Bleeding in several sites have been reported (gastrointestinal tract, brain, urogenital system, uterus, liver, gallbladder, eyes) depending on therapy's intensity, patient's age and the base illness, but not on the treatment's duration (16). It has been also reported a $32 \%$ of haemorrhages with warfarin and an $11 \%$ with acenocoumarol (16). Mechanisms related with acenocoumarol's interactions are absorption's disorders, inhibition or induction of the metabolizing enzymatic system and a reduced vitamin $\mathrm{K}$ availability. It is necessary to look close after coagulation and to control it often when other drugs are prescribed acenocoumarol or when simultaneous intake is stopped (17). We are advised against giving acenocoumarol with other drugs which modify the hemostasis in order not to increase the bleeding risk (heparin, acetylsalicylic acid and derivatives, phenylbutazone and other pyrazolonic derivatives). When combining acenocoumarol with NSAIDs, it is recommended to check more often the coagulation (17).

In our series, a variation on the withdrawal standard was found, varying between 3 and 7 days prior to surgery. No differences in the transfusion requirements between GA and GB were found. A case of acenocoumarol and ASA interaction was recorded, which required the urgent transfusion of ten bags with packed red blood cells in a female patient who underwent a cystocele correction plus REMEEX system placement for complex urinary incontinence, requiring an endopelvic packing to save the patient's life (18).

\section{Flavonoids}

They are an aromatic group of heterocyclic pigments with anti-allergic, anti-inflammatory, antioxidant, anti-microbial (antibacterial, antifungal, and antiviral, anti-cancer, anti-thrombotic and anti-diarrheal activities (19). The treatment with flavonoids is not exempt from pharmacological interactions. In some cases they can inactivate certain isoenzymes of the cytochrome P450 (3A4 and 2C9) or inhibit the hepatic glucuronosyltransferases (20). We usually recommend to stop the intake of any flavonoids 7 10 days prior to surgery, suggested by the Urologist.

\section{Triflusal}

It is a platelet aggregation inhibitor of the salicylate family but it is not a derivative of ASA, effective in the prevention of cardiovascular events (stroke, acute treatment of cerebral infarction, myocardial infarction) but with less gastrointestinal effects than ASA (stomach haemorrhage and peptic ulcer) (21). Less than a $1 \%$ of haemorrhagic events have been reported (22). Additive effects can be observed with AC/AP drugs, NSAIDs and thrombolytic drugs. High doses of salycilates (> 6 g/day) can also develop an hypoprothrombinemia, and additional bleeding risk factor (23).

\section{Pentoxifylline}

It is a semisynthetic dimethylxanthine derivative with haematologic effects (24). Its plasmatic concentrations are increased with concomitant ciprofloxacin (the quinolone reduces the hepatic metabolism of the pentoxifylline and increases the erythrocytic metabolism) (25). Cimetidine, an inhibitor of the hepatic enzymes, can also increase the pentoxifylline's plasmatic concentrations (25). It has been reported an increase of the prothrombin time when using pentoxifylline and warfarin at the same time (25). It is recommended to monitor prothrombin time, haemoglobin and haematocrit (25). The simultaneous administration of antiplatelet drugs and pentoxifylline has not been studied, so special attention to synergism must be paid (25). In a small number of patients stabilized with theophylline, pentoxifylline's administration increased its plasmatic level with the subsequent toxicity risk. It should be noted that both drugs are xanthine derivatives and no cross-reactions are described (25). As shown in Table 3, there are no patients treated with enoxaparin, pentoxifylline, bemiparin, diosminthesperidin derivatives or triflusal in GB. Although there are no significant differences, it is possible that in other series with larger $\mathrm{n}$ the explanation to this difference could be found.

More patients with high grade $(p=0.003)$ and higher $T$ stage $(p=0.0018)$ tumours were found in GA after a VTUR due to BTCC, regardless of wether AC/AP drugs were previously taken.

\section{Conclusions}

The pathologies which most needed blood components' transfusions in the Urology Department were (in order of frequency): bladder cancer, kidney cancer, prostate cancer, prostate adenoma, other urological diagnoses.

ASA $100 \mathrm{mg}$ did not influence the transfusion's requirements in VTUR due to BTCC. Tumour stage and higher grade have a greater influence in transfusion's requirements than concomitant AP/AC treatment. The heterogeneity of AP/AC protocols does not allow to establish the benefit of stopping those drugs before surgery in terms of avoiding blood transfusions when performing a VTUR.

\section{References}

1. Lozano-Sanchez F. Valoración de la eficacia en la prevención de la enfermedad tromboembólica venosa en pacientes médicos y quirúrgicos, in Enfermedad tromboembólica venosa, E. RochaHernando and R. Lecumberri-Villamediana, Editors. 2012, Grupo Acción Médica: Madrid. p. 105-110.

2. Karkouti $K$, et al. The independent association of massive blood loss with mortality in cardiac surgery. Transfusion. 2004; 44:1453-62. 
3. Paramo J, et al., Fundamentos básicos para el empleo de hemoderivados y estrategias de ahorro de sangre en cirugía. Rev Med Univ Navarra. 2008; 52:9-14.

4. FitzGerald GA, Patrono C. The coxibs, selective inhibitors of cyclooxygenase-2. N Engl J Med. 2001; 345:433-42.

5. Flower RJ. The development of COX2 inhibitors. Nat Rev Drug Discov. 2003; 2:179-91.

6. Hawkey CJ. COX-2 inhibitors. Lancet. 1999; 353:307-14.

7. Patrono $C$, et al. Platelet-active drugs: the relationships among dose, effectiveness, and side effects: the Seventh ACCP Conference on Antithrombotic and Thrombolytic Therapy. Chest 2004; $126(3$ Suppl):234s-264s.

8. Ware JA, Heistad DD. Seminars in medicine of the Beth Israel Hospital, Boston. Platelet-endothelium interactions. N Engl J Med. 1993; 328:628-35.

9. Hawkey C Gastrointestinal toxicity of non steroids anti-inflammatory drugs, in Therapeutic toles of selective COX2 inhibitors, J. Vane and R. Botting, Editors. 2001, William Harvey Press: Londres. p. 355-394.

10. Kunapuli SP, Daniel JL P2 receptor subtypes in the cardiovascular system. Biochem J. 1998; 336 (Pt 3):513-23.

11. CAPRIE.Steering.Committee, A randomised, blinded, trial of clopidogrel versus aspirin in patients at risk of ischaemic events (CAPRIE). CAPRIE Steering Committee. Lancet. 1996; 348:1329-39.

12. Mills DC, et al. Clopidogrel inhibits the binding of ADP analogues to the receptor mediating inhibition of platelet adenylate cyclase. Arterioscler Thromb, 1992; 12:p. 430-6.

13. Caplain H, D'Honneur G, Cariou R. Prolonged heparin administration during clopidogrel treatment in healthy subjects. Semin Thromb Hemost. 1999; 25 Suppl 2:61-4.

14. Kamath S, et al. A prospective randomized trial of aspirin-clopidogrel combination therapy and dose-adjusted warfarin on indices of thrombogenesis and platelet activation in atrial fibrillation. J Am Coll Cardiol. 2002; 40:484-90.

15. Barcellona D, et al. Warfarin or acenocoumarol: which is better in the management of oral anticoagulants? Thromb Haemost. 1998; 80:899-902.

16. Amian A, et al. Comparative study of the stability of oral anticoagulant treatments (warfarin vs acenocoumarol). Sangre (Barc). 1996; 41:9-11.

17. Myers S. Interactions between complementary medicines and warfarin. Australian Prescriber. 2002;25:54-56.

18. Padilla-Fernández B, et al. Complicaciones graves y fracasos de la corrección quirúrgica de la incontinencia urinaria de esfuerzo mediante prótesis Remeex. Aportación de nuestra serie 45 casos., in XII Congreso SINUG (Sociedad Iberoamericana de Neurourología y Uroginecología). S.I.d.N.y. Uroginecología, Editor. 2012: Cádiz. España.

19. Sax N, Lewis R. Diccionario de química y de productos químicos. 2 ed ed, ed. hAWLEY. 1993, Barcelona:oMEGA.

20. Prasain JK, Carlson SH, Wyss JM. Flavonoids and age-related disease:risk, benefits and critical windows. Maturitas. 2010;. 66:163-71.

21. Matias-Guiu J, et al. Comparison of triflusal and aspirin for prevention of vascular events in patients after cerebral infarction: the TACIP Study: a randomized, double-blind, multicenter trial. Stroke. 2003; 34:840-8.

22. Sanchez de Miguel L, et al. The effect of triflusal on human platelet aggregation and secretion: the role of nitric oxide. Rev Esp Cardiol. 2000; 53:205-11.

23. Murdoch D, Plosker GL. Triflusal: a review of its use in cerebral infarction and myocardial infarction, and as thromboprophylaxis in atrial fibrillation. Drugs. 2006; 66:671-92.

24. Vinik AI, et al. Diabetic neuropathies. Diabetes Care. 1992; 15:1926-75.

25. Goodnan-Gilman A. Las bases farmacológicas de la terapéutica. 10 ed ed, ed. McGraw-Hill/Intramericana de Mexico 2003.

\section{Correspondence}

María Fernanda Lorenzo-Gómez (Corresponding Author)

mflorenzogo@yahoo.es

C/ Esmeralda 6. Urb Las Canteras.

Villamayor. 37185 Salamanca, Spain - 616422924

Alvaro Julio Virseda-Rodríguez, MD

Lauro Sebastian Valverde-Martinez, MD

Department of Urology. University Hospital of Salamanca.

Barbara Padilla-Fernández, MD

Department of Urology. University Hospital of the Canary Islands, Tenerife

Mirian López-Parra, MD

Maria Jesus Nieto González, MD

Department of Haematology. University Hospital of Salamanca

Maria Tatiana Santos-Antunes, MD

Anabel Lorenzo-Gómez, MD

Maria Begoña García-Cenador, MD

Department of Surgery, University of Salamanca

Jesus Fernando San Miguel-Izquierdo, MD

Clinical and Translational Medicine of the University of Navarra

Patricia Antúnez-Plaza, MD

Department of Pathology, University Hospital of Salamanca 